\title{
Fault Detection and Isolation of inland navigation channel: Application to the Cuinchy-Fontinettes reach
}

\author{
E. Duviella, L. Rajaoarisoa, J. Blesa and K. Chuquet
}

\begin{abstract}
Inland navigation networks are mainly used for transport with economic and environmental benefits. In a climate change context which leads to the scarcity of the water resource, the control of navigation levels and the supervision of these networks become crucial. Thus, this paper is focused on the sensors Fault Detection and Isolation of inland navigation reaches. A modeling method based on the identification technique is proposed. Then, based on residuals, the dynamic classification algorithm $A U D Y C$ leads to the detection and diagnosis of sensors faults. Setting errors and slow drifts are considered. The proposed methods are applied on the CuinchyFontinettes reach benchmark.
\end{abstract}

\section{INTRODUCTION}

Most of the inland navigation networks in the north of Europe have been built and arranged by human to improve the navigation. They are composed of reaches or channels separated by locks, and can be supplied by water volumes stocked in dams. The control of the navigation channels consists in maintening the seaworthiness requirements, in particular, the water levels of the navigation channels [4]. This aim is more important in a climate change context where the use of water has to be the most efficient as possible. Automatic control provides accuracy and reliability if no sensor or actuator fault occurs. Otherwise, sensor or actuator faults lead to serious dysfunctions. In particular, sensors are subjected to setting errors, transmission faults, or drifts of operation. Their occurence can impact navigation, infrastructures, and available water resources. Thus, Fault Detection and Isolation (FDI) techniques have to be developped.

FDI techniques require accurate models of the system dynamic. Based to these models, the operating modes of the systems can be characterized, and the faults can be detected and diagnosed. In the litterature, a large number of FDI techniques are proposed. Model-based approaches were proposed [8], based on parameters identification technique [23], parity equations method [9], diagnosis observers [1], or Kalman filters [24]. In the field of hydraulic systems, fault detection methods based on residual generation, extended Kalman filter and finite memory observer are proposed in [3]. The objective was to detect and localize leak in an irrigation

This work is a contribution to the GEPET-Eau project which is granted in the framework of the GICC program of the French Ministry MEDDE, with the participation of the CGDD, the DGEC and the DGTIM.

E. Duviella and L. Rajaoarisoa are with Univ Lille Nord de France - MinesDouai, IA, France \{eric.duviella, lala.rajaoarisoa\} @mines-douai.fr

J. Blesa is with the Automatic Control Department, Universitat Politecnica de Catalunya, Barcelona, Spain joaquim.blesa@upc.ed

K. Chuquet is with VNF - Service de la navigation du Nord Pas-de-Calais, Lille, France karine.chuquet@developpement-durable.gouv. fr network. In [11], a FDI technique based on the dynamical classification algorithm $A U D y C$ [10], is proposed for the detection of faults of a navigation channel. In this paper, FDI based on classification approaches appears adapted to the fault detection of large scale hydraulic systems without model. However, the proposed approach considers only data from sensors. The controled inputs of the system, i.e. controlled gates and locks, are not taken into account. In [12], the proposed sensor fault detection techniques are focused on a real undershot/overshot gate. They are based on physical and nonlinear black-box models. Very recently in [2], an online strategy for sensor and/or actuator FDI is applied to a dam-gallery. This strategy consists in a recursive subspace identification algorithm to estimate the dam-gallery model parameters. Then, a fault estimation procedure is proposed to detect potential faults. The proposed method is suitable for open channel systems with the assumption of constant faults on a minimim time window.

This paper aims at proposing a FDI strategy for sensors of inland navigation reaches, with an application on the Cuinchy-Fontinettes Reach (CFR) which is located in the north of France. The FDI strategy is based on a gray-box modeling approach for multi-inputs-multi-ouputs (MIMO) and time-delay open-channel systems [7]. Gray box modeling is a popular approach for modeling real systems, combining both black box and white box methodologies [21]. Herein, the use of gray-box model is justified by the characteristics of inland navigation reaches; long length (several kilometers), no slope, wave phenomena, etc. Indeed, the well-known Saint Venant equations [6] can not be applied without requiring numeric models. Moreover, the modeling techniques based on the simplification and linearization of the Saint-Venant equations [14], are not dedicated for noslope channels characterized by wave phenomenas. Finally, the modeling approach proposed in [18] is not dedicated for very long channels.

Then, based on the measures of the real system and on the outputs of the gray-box model, residuals are generated. They are used for the detection and the diagnosis of setting errors and slow drifts on sensors. To achieve this aim, the proposed FDI technique is based on classification methods. An overview of the classification methods for the diagnosis of dynamical systems is proposed in [19]. Among these classification techniques, the dynamical classification algorithm $A U D y C$ is selected. This algorithm is particularly well adapted for the FDI of dynamical evolving systems as it is shown in [5], [11], [22].

The paper is organized as following: Section II is ded- 


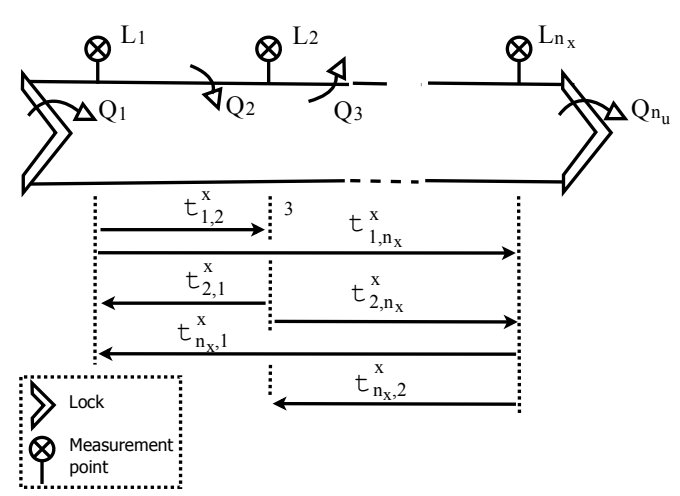

Fig. 1. Time delays $\tau_{i, j}^{x}$ between each measurement points.

icated to the description of the gray box modeling approach for inland navigation reaches. In Section III, the FDI technique based on the $A U D y C$ algorithm is presented. The modelling of the CFR is described in Section IV. The effectiveness of the proposed FDI approach is highlighted in Section $\mathrm{V}$ by considering two scenarios of faults.

\section{MODELING OF INLAND NAVIGATION REACH}

Inland navigation networks are composed of interconnected reaches generally with no significant slope and MIMO. A navigation reach is an open-channel system characterized by large dimensions, and nonlinear dynamics with varying delays. The navigation being assured by the control of the water level with the objective to be close to the Normal Navigation Level (NNL), an assumption of linearity can be realized. The proposed model is a gray-box linear state-space model (see [7]).

The state variables are chosen as the $n_{x}$ measured levels on the system, i.e. $L_{i}(k)$. The input variables correspond to the $n_{u}$ input/output discharges $Q_{l}(k)$ (see Figure 1). The output variables correspond directly to the state variables. Finally, time delay matrices $\tau^{x}$ and $\tau^{u}$ are considered for each state and input variables to take into account the transfer delays between two points of the reach.

$$
\left\{\begin{array}{l}
x_{k+1}=A \bar{x}_{k \mid \tau^{x}}+B \bar{u}_{k \mid \tau^{u}} \\
y_{k}=C x_{k}
\end{array}\right.
$$

where matrices $A \in \mathbb{R}^{n_{x} \times n_{x} \cdot n_{x}}, B \in \mathbb{R}^{n_{x} \times n_{u} \cdot n_{x}}$ and $C \in \mathbb{R}^{n_{x} \times n_{x}}$ are the state, input and output matrices, respectively. At each time $k$, the vector $\bar{u}_{k \mid \tau_{i}^{u}} \in \mathbb{R}^{n_{u} \cdot n_{x}}$ representing the input variable is defined according to the delay matrix $\tau^{u}$, the vector $\bar{x}_{k \mid \tau^{x}} \in \mathbb{R}^{n_{x} . n_{x}}$ representing the state variable according to the delay matrix $\tau^{x}$. The matrices $\tau^{x} \in \mathbb{N}^{n_{x} \times n_{x}}$ and $\tau^{u} \in \mathbb{N}^{n_{u} \times n_{x}}$ gather the time delays between each measurement point (see Figure 1), and between each measurement point and each input and output of the system, respectively. For example, the value of $\tau_{i, j}^{x} \in \mathbb{N}$ is the time delay between the measurement points
$L_{i}$ and $L_{j}$

$$
\tau^{x}=\left[\begin{array}{cccc}
\tau_{1,1}^{x} & \tau_{1,2}^{x} & \cdots & \tau_{1, n_{x}}^{x} \\
\tau_{2,1}^{x} & \tau_{2,2}^{x} & \cdots & \tau_{2, n_{x}}^{x} \\
\vdots & \vdots & \ddots & \vdots \\
\tau_{n_{x}, 1}^{x} & \tau_{n_{x}, 2}^{x} & \cdots & \tau_{n_{x}, n_{x}}^{x}
\end{array}\right]
$$

The time delays $\tau_{i, j}^{x}$ are equal to 1 for $i=j$. The time delay matrices $\tau^{x}$ and $\tau^{u}$ are supposed to be known and constant. The vector $y_{k} \in \mathbb{R}^{n_{x}}$ represents the output variable of the system.

The elements of the matrices $\tau^{x}$ and $\tau^{u}$ are obtained by a correlation method (data-based procedure) or by the physical knowledge of the system. Consider two points along the canal, separated by a distance $D$ : upstream and downstream points. According to [13], the theoretical value of the upstream time delay between these two points is evaluated by computing the integral:

$$
\tau_{u p}=\int_{0}^{D} \frac{d l}{c(l)+v(l)}
$$

with $c(l)$ and $v(l)$ representing the celerity and the velocity respectively.

This corresponds to the minimum time required for a perturbation to travel from the upstream point to the downstream point. Analogously, the downstream time delay $\tau_{d w}$ can be evaluated by:

$$
\tau_{d w}=\int_{0}^{D} \frac{d l}{c(l)-v(l)}
$$

and corresponds to the maximum time required for a perturbation to travel from the upstream point to the downstream point. In both cases, we recover the classical value in the uniform case when $v$ and $c$ are constant: $\tau_{u p}=\frac{D}{c+v}$ and $\tau_{d w}=\frac{D}{c-v}$, with $c=\sqrt{g \frac{S}{b}}$ and $v=\frac{Q}{S}$. The wet aeras $S$ and the cross section $b$ are chosen constant as the mean values, $g$ is the gravity and $Q$ is the average flow of the canal.

The vector $x_{k}$ in (1) is:

$$
x_{k}=\left[\begin{array}{llll}
L_{1}(k) & L_{2}(k) & \cdots & L_{n_{x}}(k)
\end{array}\right]^{T}
$$

Thus, the vector $\bar{x}_{k \mid \tau^{x}}$ is built according to the matrix $\tau^{x}$ such as:

$$
\begin{aligned}
& \bar{x}_{k \mid \tau^{x}}=\left[L_{1}\left(k-\tau_{1,1}^{x}\right) L_{2}\left(k-\tau_{1,2}^{x}\right) \cdots L_{n_{x}}\left(k-\tau_{1, n_{x}}^{x}\right)\right. \\
& L_{1}\left(k-\tau_{2,1}^{x}\right) L_{2}\left(k-\tau_{2,2}^{x}\right) \cdots L_{n_{x}}\left(k-\tau_{2, n_{x}}^{x}\right) \\
& \left.\cdots L_{n_{x}}\left(k-\tau_{n_{x}, n_{x}}^{x}\right)\right]^{T}
\end{aligned}
$$

The vector $\bar{u}_{k \mid \tau^{u}}$ is built according to the matrix $\tau^{u}$ similarly as relation (6):

$$
\begin{aligned}
\bar{u}_{k \mid \tau^{u}}= & {\left[Q_{1}\left(k-\tau_{1,1}^{u}\right) Q_{2}\left(k-\tau_{1,2}^{u}\right) \cdots Q_{n_{u}}\left(k-\tau_{1, n_{u}}^{u}\right)\right.} \\
& Q_{1}\left(k-\tau_{2,1}^{u}\right) Q_{2}\left(k-\tau_{2,2}^{u}\right) \cdots Q_{n_{u}}\left(k-\tau_{2, n_{u}}^{u}\right) \\
& \left.\cdots Q_{n_{u}}\left(k-\tau_{n_{x}, n_{u}}^{u}\right)\right]^{T}
\end{aligned}
$$


The state, input and ouput matrices are defined as:

$$
\begin{aligned}
A & =\left[\begin{array}{cccccccccc}
a_{1}^{1} & a_{2}^{1} & \cdots & a_{n_{x}}^{1} & 0 & 0 & \cdots & 0 & \cdots & 0 \\
0 & 0 & \cdots & 0 & a_{1}^{2} & a_{2}^{2} & \cdots & a_{n_{x}}^{2} & \cdots & 0 \\
\vdots & \vdots & \ddots & \vdots & \vdots & \vdots & \ddots & \vdots & \ddots & \vdots \\
0 & 0 & \cdots & 0 & 0 & 0 & \cdots & 0 & \cdots & a_{n_{x}}^{n_{x}}
\end{array}\right] \\
B & =\left[\begin{array}{cccccccccc}
b_{1}^{1} & b_{2}^{1} & \cdots & b_{n_{u}}^{1} & 0 & 0 & \cdots & 0 & \cdots & 0 \\
0 & 0 & \cdots & 0 & b_{1}^{2} & b_{2}^{2} & \cdots & b_{n_{u}}^{2} & \cdots & 0 \\
\vdots & \vdots & \ddots & \vdots & \vdots & \vdots & \ddots & \vdots & \ddots & \vdots \\
0 & 0 & \cdots & 0 & 0 & 0 & \cdots & 0 & \cdots & b_{n_{u}}^{n_{u}}
\end{array}\right]
\end{aligned}
$$

The matrix $C$ is equal to the identity matrix of order $n_{x}$. After the structure of the state-space model has been defined, the objective is to identify the matrices $A$ and $B$. Model (1) can be rewritten as:

$$
x_{k+1}=M \Phi_{k}
$$

with $M=\left[\begin{array}{ll}A & B\end{array}\right]$ and $\Phi_{k}=\left[\begin{array}{ll}\bar{x}_{k \mid \tau^{x}} & \bar{u}_{k \mid \tau^{u}}\end{array}\right]^{T}$.

Then, the matrix $M$ has to be determined according to an identification approach using available measured data. The data correspond to $N$ samples of the discharges $Q_{i}$ and levels $L_{i}$ measured on a time interval. Based on relation (10), the matrix $M$ is expressed in the following way:

$$
M=X \bar{\Phi}^{T}\left(\bar{\Phi} \bar{\Phi}^{T}\right)^{-1}
$$

Considering the characteristics of the matrices $A$ and $B$, and the size of $X$ and $\bar{\Phi}$, it is easier to identify separatly each line of $M$, before rebuilding the global matrices $A$ and $B$. The zeros of matrices $A$ and $B$ are not considered during the identification step. It is based on the principle that each state variable can be expressed such as the first state variable:

$$
\begin{aligned}
& L_{1}(k+1)=a_{1}^{1} L_{1}\left(k-\tau_{1,1}^{x}\right)+a_{2}^{1} L_{2}\left(k-\tau_{1,2}^{x}\right)+\cdots \\
& +a_{n_{x}}^{1} L_{n_{x}}\left(k-\tau_{1, n_{x}}^{x}\right)+b_{1}^{1} Q_{1}\left(k-\tau_{1,1}^{u}\right) \\
& +b_{2}^{1} Q_{2}\left(k-\tau_{1,2}^{u}\right)+\cdots+b_{n_{u}}^{1} Q_{n_{u}}\left(k-\tau_{1, n_{u}}^{u}\right)
\end{aligned}
$$

Using this particular structure of the state space model, the dynamics of a real navigation reach can be identified. Then, this gray-box model is used to detect and isolate sensor faults thanks to the $A U D y C$ algorithm.

\section{FDI BASED ON AUDYC}

The proposed FDI method is based on the classification algorithm $A U D y C$. This classification algorithm consists in characterizing on-line the operating mode of the system by a Gaussian model which constitute a class. A class is determined according to pertinent indicators, i.e. the features. According to the features, a Representation Space can be built, and the class can be represented in this space. Thus, the class of the normal operating mode, denoted $C_{n}$, can be determined (see Figure 2). A new class is create when a sufficient number of points is present in an area of the Representation Space. The new class, which is updated or created, is denoted evolutionary class $C_{k}$. It corresponds to a new operating mode. When an aberrant measurement occurs, a new point appears in the Representation Space far from the current class $C_{k}$. This point is detected and rejected.

8) Based on these functionalities, sudden changes (jump in the Representation Space) or progressive changes of the current operating mode can be detected. When several observations are not affected to the current class, a new class $C_{k}$ is built and a sudden change is detected (see Figure 2.a). The on-line updating of the characteristics of the current class allows the detection of drift (see Figure 2.b).
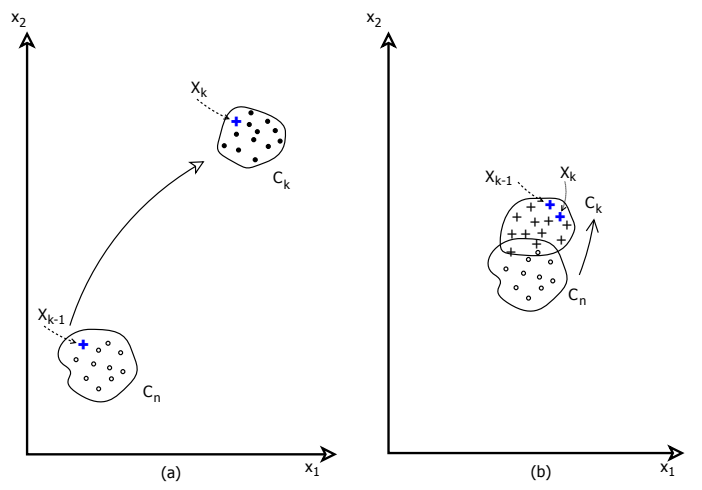

Fig. 2. Different types of faults in a Representation Space; (a) jump and (b) drift.

Operating modes are Gaussian classes. Each class $C^{j}$ is characterized by a center and a covariance matrix. These parameters are adapted on- line using rules of recursive update. The updated parameters of the classes is performed recursively on a sliding window size $N_{f e n}$ for each new observation $X_{k}=\left(x_{1}, x_{2}, \ldots x_{n}\right)$, with $n$ the number of features. The principle of the procedure for adjusting the class is given by:

- If $\operatorname{Card}\left(C^{j}\right)=n_{c}<N_{f e n}$ : add information

$$
\left\{\begin{aligned}
M_{C^{j}}(k) & =M_{C^{j}}(k-1)+\frac{1}{n_{c}+1}\left(X_{k}-M_{C^{j}}(k-1)\right), \\
\Omega_{P^{j}}(k) & =\frac{n_{c}-1}{n_{c}} \Omega_{C^{j}}(k-1)+ \\
& \frac{1}{n_{c}+1}\left(X_{k}-M_{C^{j}}(k-1)\right)^{\top}\left(X_{k}-M_{C^{j}}(k-1)\right) .
\end{aligned}\right.
$$

- If $n_{c} \geq N_{f e n}$ : add and Removing information

$$
\begin{aligned}
& \left\{\begin{aligned}
& M_{C^{j}}(k)=M_{C^{j}}(k-1)+\frac{1}{N_{f e n}}\left(\delta X^{+}-\delta X^{-}\right), \\
& \Omega_{C^{j}}(k)=\Omega_{C^{j}}(k-1)+ \\
& \Delta X\left[\begin{array}{cc}
\frac{1}{N_{f e n}} & \frac{1}{N_{f e n}\left(N_{f e n}-1\right)} \\
\frac{1}{N_{f e n}\left(N_{f e n}-1\right)} & -\frac{\left(N_{f e n}+1\right)}{N_{f e n}\left(N_{f e n}-1\right)}
\end{array}\right] \Delta X^{\top},
\end{aligned}\right. \\
& \text { where } \\
& \left\{\begin{array}{c}
\delta X^{+}=X^{\text {new }}-M_{C^{j}}(k-1), \\
\delta X^{-}=X^{\text {old }}-M_{C^{j}}(k-1), \\
\Delta X=\left[\delta X^{+} \delta X^{-}\right],
\end{array}\right.
\end{aligned}
$$

with $M_{C^{j}}(k)$ and $\Omega_{C^{j}}(k)$ respectively the center and the covariance matrix of the class $C^{j}$ at instant $k, X^{n e w}$ and $X^{\text {old }}$ new and old observation vectors, respectively. 


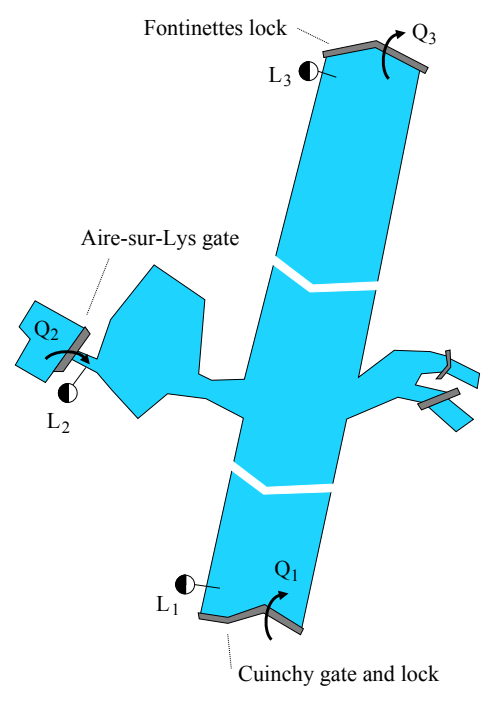

Fig. 3. Scheme of the Cuinchy-Fontinettes navigation reach.

Fault indicator is calculated for the detection of faults. It consists in computing the distance between the evolutive class $C_{k}$ and the normal class $C_{n}$. The normal class is taken as reference. A fault occurence can lead to the drift of the evolutive class $C_{k}$ from the normal class $C_{n}$. Amongst the several existing metrics, the Euclidian distance, denoted $d\left(M_{k}, M_{n}\right)$, is considered:

$$
d\left(M_{k}, M_{n}\right)=\sqrt{\left(M_{k}-M_{n}\right)\left(M_{k}-M_{n}\right)^{\top}},
$$

with $M_{k}$ is the center of the class $C_{k}$ and $M_{n}$ the center of the normal class $C_{n}$. The center of the normal class $M_{n}$ is fixed.

Finally, a fault will be detected when a prefixed threshold th is crossed. This threshold is tuned according to expert knowledge on the system.

\section{CUINCY-FONTINETTES REACH GRAY-BOX MODEL}

\section{A. Presentation}

The Cuinchy-Fontinettes navigation reach (CFR) is part of the broad gauge river network of North of France. It is located between the upstream lock of Cuinchy at the East of the town Bethune and the downstream lock of Fontinettes at the Southwest of the town Saint-Omer (see Figure 3). The first part of the channel from Cuinchy to Aire-sur-la-Lys, is $28.7 \mathrm{~km}$ long. The second part of the channel from Airesur-la-Lys to Saint-Omer is $13.6 \mathrm{~km}$ long. The channel is entirely artificial with a bottom equal to $52 \mathrm{~m}$ in average and no significant slope. Considering the navigation flow, the water runs off from Cuinchy to Fontinettes.

The CFR is managed by VNF (Voies Navigables de France) whose role is to maintain the level of the channel at $N N L=4.26 \mathrm{~m}$. To reach this aim, three points of the CFR must be controlled: the first is the Cuinchy lock and gate, the second is the Fontinettes lock and the third is the gate called "Porte de Garde" at Aire-sur-la-Lys. The control of Cuinchy and Fontinettes locks is constrained by the navigation demand. The size of the lock at Fontinettes is $13 m$ high whereas the lock at Cuinchy is only $2 m$ high. The operation of the Fontinettes lock causes a wave phenomena which impact the CFR during more than 2 hours with a maximum amplitude which exceeds $13 \mathrm{~cm}$. The sensors of the CFR are, as every electronic device, subject to faults. These sensors can break down or be impacted over time. Several types of errors on sensors can occur. A bad setting of the sensors can lead to constant errors. To detect and isolate these sensor faults, the gray-box model of the CFR is achieved.

\section{B. Gray-box modeling}

The CFR has three inputs, $Q_{1}$ for Cuinchy, $Q_{2}$ for Airesur-la-Lys, and $Q_{3}$ for Fontinettes, i.e. $n_{u}=3$. The levels of the CFR are measured on three points; $L_{1}$ at Cuinchy, $L_{2}$ at Aire-sur-la-lys and $L_{3}$ at Fontinettes, i.e. $n_{x}=3$. The time-delays are determined according to relations (3) and (4), with the wet aeras $S=52 \times 4.26 \mathrm{~m}^{2}$ (bottom $\times$ level of the water), and the maximum discharge $Q=7.6 \mathrm{~m}^{3} / \mathrm{s}$ which is evaluated according to the Fontinettes lock operation. The matrices $\tau^{x}=\tau^{u}$ are given in minutes:

$$
\tau^{x}=\left[\begin{array}{ccc}
1 & 74 & 109 \\
75 & 1 & 35 \\
110 & 36 & 1
\end{array}\right]
$$

The vector $x_{k}$ is $x_{k}=\left[L_{1}(k) L_{2}(k) L_{3}(k)\right]^{T}$. The output matrix is equal to $C=I_{3}$.

The modeling approach consists in identifying the coefficients of the state and input matrices defined as:

$$
\begin{gathered}
A=\left[\begin{array}{ccccccccc}
a_{1}^{1} & a_{2}^{1} & a_{3}^{1} & 0 & 0 & 0 & 0 & 0 & 0 \\
0 & 0 & 0 & a_{1}^{2} & a_{2}^{2} & a_{3}^{2} & 0 & 0 & 0 \\
0 & 0 & 0 & 0 & 0 & 0 & a_{1}^{3} & a_{2}^{3} & a_{3}^{3}
\end{array}\right] \\
B=\left[\begin{array}{ccccccccc}
b_{1}^{1} & b_{2}^{1} & b_{3}^{1} & 0 & 0 & 0 & 0 & 0 & 0 \\
0 & 0 & 0 & b_{1}^{2} & b_{2}^{2} & b_{3}^{2} & 0 & 0 & 0 \\
0 & 0 & 0 & 0 & 0 & 0 & b_{1}^{3} & b_{2}^{3} & b_{3}^{3}
\end{array}\right]
\end{gathered}
$$

\section{Identification of the CFR model}

The identification task consists in estimating the coefficients of the matrices $A$ and $B$ according to equations (11). The step is twofold: (i) estimation of the parameters on a first scenario, (ii) evaluation of the model on other several scenarios. This step has been achieved in [7]. Thus the identification step is summarized herein.

The CFR is modeled using the Saint-Venant equations via the software SIC [17]. A mean rectangular profile is considered with a bottom equal to $S=52 \mathrm{~m}$, a length equal to $L=42300 \mathrm{~m}$ and a quasi-nul slope equal to $2.4 e^{-5} \%$. Then, the actuators of the CFR are modeled using the software Matlab/Simulink. A simulation architecture coupling SIC and Matlab/Simulink allows the resolution of the Saint-Venant equations according to scenarios defined on Matlab/Simulink. These scenarios consist in the simulation of the operation of the locks and gates of the CFR. The 

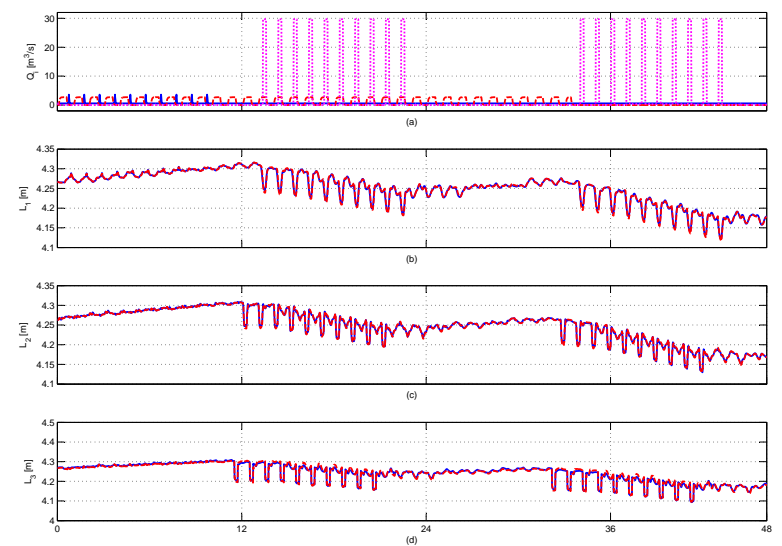

Fig. 4. (a) Discharges at Cuinchy $Q_{1}$ (blue continuous line), at Aire-surla-Lys $Q_{2}$ (red dotted line) and at Fontinettes $Q_{3}$ (magenta dashed line), levels, estimated (red dashed line) and measured (blue continuous line) at (b) Cuinchy $L_{1}$, (c) Aire-sur-la-Lys $L_{2}$ and (d) Fontinettes $L_{3}$.

TABLE I

CoEfFicients of the MATRICES $A\left(\times e^{-1}\right)$ AND $B\left(\times e^{-3}\right)$.

\begin{tabular}{|c|c|c|c|c|c|c|c|c|}
\hline$a_{1}^{1}$ & $a_{2}^{1}$ & $a_{3}^{1}$ & $a_{1}^{2}$ & $a_{2}^{2}$ & $a_{3}^{2}$ & $a_{1}^{3}$ & $a_{2}^{3}$ & $a_{3}^{3}$ \\
\hline 6.77 & 2.61 & 0.61 & 2.73 & 3.9 & 3.34 & 4.08 & 3.88 & 2.04 \\
\hline$b_{1}^{1}$ & $b_{2}^{1}$ & $b_{3}^{1}$ & $b_{1}^{2}$ & $b_{2}^{2}$ & $b_{3}^{2}$ & $b_{1}^{3}$ & $b_{2}^{3}$ & $b_{3}^{3}$ \\
\hline 0.4 & 1.2 & 0.2 & 0.3 & 1 & 0.3 & 0.6 & 1.3 & 2.8 \\
\hline
\end{tabular}

scenario which is used for the identification of the matrices $A$ and $B$ corresponds to two days of navigation, with a positive discharge at Cuinchy equal to $0.6 \mathrm{~m}^{3} / \mathrm{s}$ and a discharge at Fontinettes equal to zero. The discharge at Aire-sur-la-Lys is considered such as a pulse signal with a frequency of $2.7 e^{-4}$ $\mathrm{Hz}$ and a maximum amplitude of $2.6 \mathrm{~m}^{3} / \mathrm{s}$ from time 0 to 34 hours and equal to zero thereafter (see Figure 4.a). It allows the water supply of the CFR to keep the level close to the NNL. During these two days, the increase of the CFR volume is due to 10 ships which have crossed the Cuinchy lock the first 10 hours and to the supplying flow of Airesur-la-Lys. The decrease of the CFR volume is due to the 20 ships crossing the Fontinettes lock. The simulation of the operating of the Cuinchy lock corresponds to a volume of $3700 \mathrm{~m}^{3}$ during 6 minutes with a trapezoidal profile, and a maximum discharge equal to $10.8 \mathrm{~m}^{3} / \mathrm{s}$. The simulation of the operating of the Fontinettes lock corresponds to a volume of $25000 \mathrm{~m}^{3}$ during 15 minutes with a trapezoidal profile, and a maximum discharge equal to $29 \mathrm{~m}^{3} / \mathrm{s}$.

These signals obtained according to the SIC/MATLAB/Simulink architecture are used to identify the model of the CFR. Due to the properties of the signals, the matrix $\left(\bar{\Phi} \bar{\Phi}^{T}\right)$ of the equation (11) can be singular. Thus it is often necessary to use the Moore-Penrose pseudoinverse of this matrix. Finally, the coefficients of the matrices $A$ and $B$ are given in Table I.

The outputs of the model $\hat{y}(k)$ and the measured levels $y(k)$ are compared as depicted in Figure 4. The estimated ouputs in red dashed line are very close to the measured levels in blue continuous line. To estimate the effectiveness of the model, a fitting indicator (FIT) is defined as FIT =
TABLE II

FIT INDICATORS (\%).

\begin{tabular}{|c|c|c|}
\hline$F I T_{L_{1}}$ & $F I T_{L_{2}}$ & $F I T_{L_{3}}$ \\
\hline 93 & 93 & 87 \\
\hline
\end{tabular}

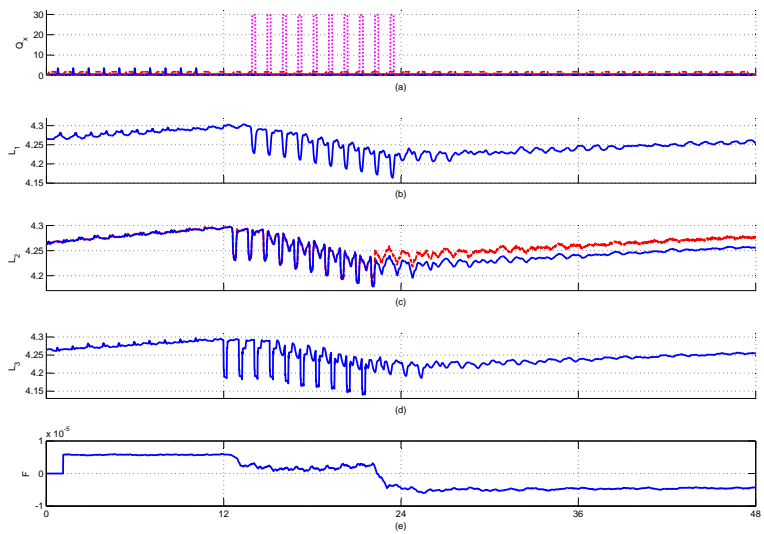

Fig. 5. Scenario 1 - (a) Discharges in Cuinchy (blue continuous line), in Aire-sur-la-Lys (red dashed line) and in Fontinettes (magenta dotted line), levels at (b) Cuinchy $L_{1}$, (c) Aire-sur-la-Lys $L_{2}$ and (d) Fontinettes $L_{3}$, and distance $d\left(M_{k}, M_{n}\right)$ between classes.

$\left(1-\frac{\|\hat{y}-y\|_{2}}{\left\|y-y_{m}\right\|_{2}}\right) \times 100$, where $y_{m}$ is a column vector composed of the mean value of $y$. For this scenario, the FIT are given for each level in Table II. Then, the effectiveness of the CFR model has been evaluated and validated using sevral scenarios [7].

\section{FDI OF THE CFR}

The evaluation of the proposed FDI technique for the CFR consists in considering two scenarios. The first one, "Scenario 1", is the simulation of the crossing of ten ships in Fontinettes. The second one, "Scenario 2", is the crossing of ten ships in Cuinchy the first ten hours and ten ships in Fontinettes the last ten hours of the first day simulating the navigation of ten ships on the CFR. In Scenario 1, a setting error of $0.02 \mathrm{~m}$ occurs on the level sensor $L_{2}$ at time 24 hours (see red dashed line in Figure 5.c). In Scenario 2, a drift occurs on the level sensor $L_{1}$ starting from time 10 hours to the end of the simulation (see red dashed line in Figure 5.b).

A Representation Space is built by considering the residuals $r_{c}, r_{a}$ and $r_{f}$ between the outputs of the model $\hat{L}_{i}(k)$ and the measured levels $L_{i}(k)$. The normal class is depicted in yellow in Figure 7. The residuals from Scenario 1 are shown in blue + , those of Scenario 2 in black dots. The faulty class for Scenario 1 and Scenario 2 are in black line and green line respectively. The detection threshold $t h$ on the distance $d\left(M_{k}, M_{n}\right)$ between the current and normal classes is tuned to zero. When this threshold is crossed, a fault is detected. Thus, fault on $L_{2}$ (Scenario 1) is detected 6 minutes after its occurence. It is isolated by considering the direction of the new class, along the $r_{a}$ axe. The same step is considered for Scenario 2. The drift on sensor $L_{1}$ is detected 6 hours after its occurence, by considering the direction of the new class 

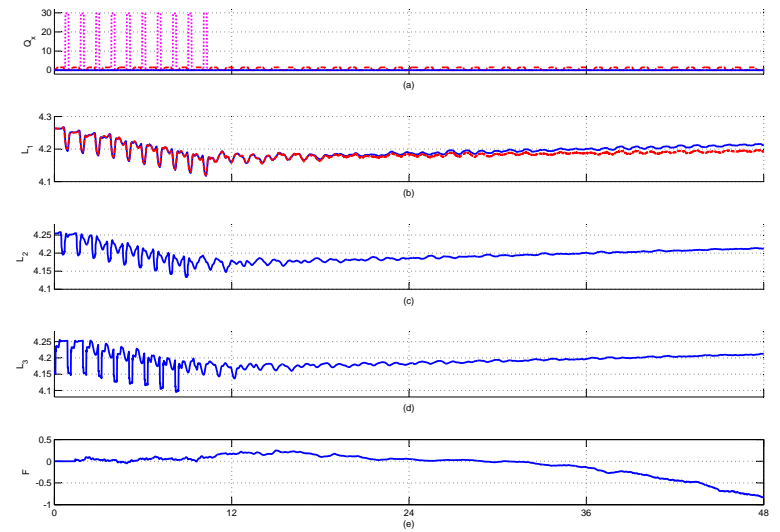

Fig. 6. Scenario 2 - (a) Discharges in Cuinchy (blue continuous line), in Aire-sur-la-Lys (red dashed line) and in Fontinettes (magenta dotted line), levels at (b) Cuinchy $L_{1}$, (c) Aire-sur-la-Lys $L_{2}$ and (d) Fontinettes $L_{3}$, and distance $d\left(M_{k}, M_{n}\right)$ between classes.

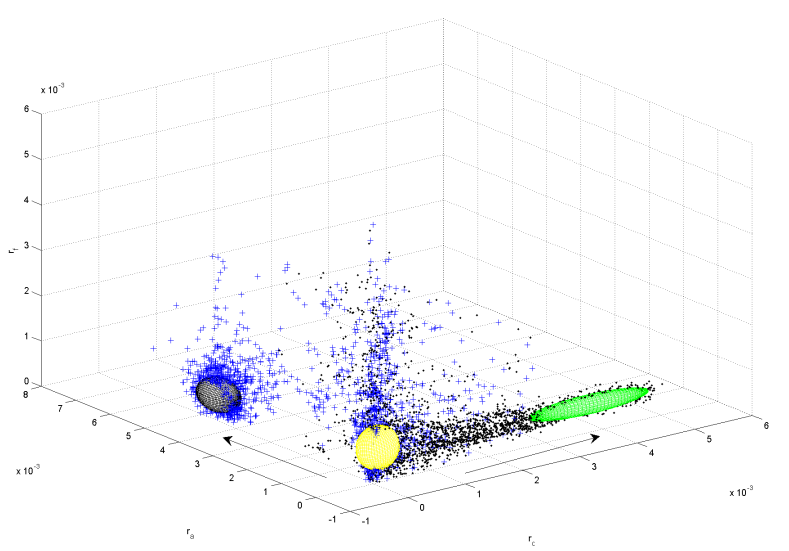

Fig. 7. Representation space according the residuals $r_{c}, r_{a}$ and $r_{f}$; normal class in yellow; for Scenario 1, residuals in blue + and faulty class in black, and for Scenario 2, residual in black dots and faulty class in green.

along the $r_{c}$ axe. In this case, the detection time appears important, but when the fault is detected, the error on the sensor is less than $10 \mathrm{~cm}$.

\section{CONCLUSION}

FDI of inland navigation channel based on a gray-box state-space model is presented in this paper. This approach is used for the Cuinchy-Fontinettes reach located in the North of France. The sensors of this reach can be impacted by sensor faults. The identification approach is performed according to data from a simulation architecture. Then, the effectiveness of the FDI technique is evaluated by considering two scenarios of fault. The future purposes consist in considering real data of the CFR.

\section{REFERENCES}

[1] Akhenak A., M. Chadli, J. Ragot and D. Maquin (2004). State estimation of uncertain multiple model with unknown inputs, 43rd IEEE Conference on Decision and Control, Atlantis, Bahamas.
[2] Akhenak A., E. Duviella, L. Bako and S. Lecoeuche (2013). On-line Fault Diagnosis using Recursive Subspace Identification Algorithm of a Dam-Gallery Open Channel System, Control Engineering Practice, In press.

[3] Bedjaoui N. and E. Weyer (2010). Algorithms for leak detection, estimation, isolation and localization in open water channels. Control Engineering Practice.

[4] J. Blesa, E. Duviella, M. Sayed-Mouchaweh, V. Puig and K. Chuquet, Automatic control to improve the seaworthiness conditions in inland navigation networks: application to a channel in the north of France, International conference on Maritime Transport, Barcelona, Spain.

[5] Chammas A., M. Traore, E. Duviella, M. Sayed-Mouchaweh and S. Lecoeuche (2012). Condition monitoring architecture for maintenance of dynamical systems with unknown failure modes, 2nd IFAC Workshop on Advanced Maintenance Engineering, Services and Technology, Seville, Espagne.

[6] V. T. Chow, (1959). Open-channel hydraulics, McGraw-Hill. New York.

[7] Duviella E., J. Blesa, L. Bako, Y. Bolea, M. Sayed-Mouchaweh, V. Puig and K. Chuquet (2013). Inland navigation channel model for fault detection and control: Application to the Cuinchy-Fontinettes reach, International Conference on Networking, Sensing and Control, ICNSC, Paris-Evry University, France.

[8] Frank P. M., S. X. Ding and B. KCipper-Seligcr (2000). Current developments in the theory of FDI. In SAFEPROCESS'00, Budapest, Hungary.

[9] Gertler J. (1998). Fault Detection and Diagnosis in Engineering Systems. Publisher: Dekker.

[10] Lecoeuche S., C. Lurette and S. Lalot (2004). New supervision architecture based on on-line modeling of non-stationary data. Neural Computing and Applications Journal, Volume 13, Pages 323-338.

[11] Le Pocher O., E. Duviella, L. Bako and K. Chuquet (2011). Sensor fault detection in a real hydraulic system using a classification approach, ICINCO'11, Noordwijkerhout, Netherlands.

[12] Le Pocher O., E. Duviella, L. Bako and K. Chuquet (2012). Sensor fault detection of a real undershot/overshot gate based on physical and nonlinear black-box models, Safeprocess'12, Mexico, Mexique.

[13] Litrico X. and V. Fromion(2004). Simplified modeling of irrigation canals for controller design. Journal of Irrigation and Drainage Engineering, Vol. 130, No. 5, pp. 373-383.

[14] Litrico X. and V. Fromion (2009). Modeling and Control of Hydrosystems, Springer.

[15] Ljung L. (1987). System identification: Theory for the user, PrenticeHall. Englewood Cliffs, N.J.

[16] Maciejowski J.M. (2002). Predictive Control: With Constraints. Prentice Hall, 2002.

[17] Malaterre P. O. (2006). SIC 5.20, Simulation of irrigation canals, http://www.cemagref.net/sic/sicgb.htm, 2006.

[18] van Overloop P.J., I.J. Miltenburg, X. Bombois, A.J. Clemmens, R.J. Strand, N.C. van de Giesen and R. Hut (2010). Identification of resonance waves in open water channels. Control Engineering Practice, Volume 18, Issue 8, Pages 863-872.

[19] Sayed Mouchaweh M. (2010). Semi-supervised classification method for dynamic applications. Fuzzy Sets and Systems, Volume 161, Issue 4, Pages 544-563.

[20] M.-C. Su and Y.-C. Liu (2005). A new approach to clustering data with arbitrary shapes. Pattern Recognition journal, Volume 38, Pages 1887-1901.

[21] Tan K., Y. Li, P. Gawthrop and A. Glidle (1997). Evolutionary GreyBox modelling for Practical Systems. Technical Report CSC-96019, Centre for Systems and Control, University of Glasgow.

[22] Traore M., E. Duviella and S. Lecoeuche (2009). Dynamical classification method to provide pertinent indicators for predictive maintenance strategies, ICINCO'09, Milan, Italia.

[23] Weihua L., R. Harigopal and S. Sirish (2003). Subspace identification of continuous time models for process fault detection and isolation. Journal of Process Control, Volume 13, Issue 5, Pages 407-421.

[24] Xie L., Y. C. Soh and C. E. de Souzi (1994). Robust kalman filtering for uncertain discrete-time systems. IEEE Transaction on Automatic Control, Volume 93, Pages 1310-1314. 